\title{
Internacionalización empresarial y sistemas de innovación en países en desarrollo
}

\author{
Isabel Álvarez \\ Departamento de Economía Aplicada II \\ e Instituto Complutense de Estudios Internacionales (ICEI) \\ Universidad Complutense de Madrid \\ Raquel Marín \\ Departamento de Economía de la Empresa, Universidad Europea de Madrid \\ e Instituto Complutense de Estudios Internacionales (ICEI)
}

\section{Resumen}

En este artículo se analiza la dinámica de la inversión directa extranjera y las formas que adopta, integrando las capacidades de I+D y la estabilidad institucional de los sistemas nacionales de innovación como factores determinantes de nuevas entradas. La estimación de un panel de datos para países con disímil nivel de desarrollo en el período 1998-2004 permite confirmar que la relevancia de los factores de atracción difiere según formas de entrada. Se comprueba el carácter acumulativo del capital extranjero en los sistemas nacionales y la diversidad de comportamientos en el grupo de economías de renta media, destacándose el potencial de las economías emergentes.

Palabras Clave: Desarrollo; Formas de entrada; Empresas multinacionales; Sistemas nacionales de innovación.

Clasificación JEL: F23; O30; O57.

\begin{abstract}
Research on foreign direct investment has focused on developed countries while the relationship with development has been dominated by a generalised view. Following a dynamic approach, we hypothesize that $R \& D$ capabilities and the stability of national systems of innovation become key driver mechanisms to improve and diversify the new entries. The empirical analysis is built upon a sample of countries with dissimilar levels of development using longitudinal data for the period 1998-2004. Our findings confirm the cumulative character of foreign capital, the differentiated paths of the developing World and the potential of emerging economies.
\end{abstract}

Keywords: Development; Entry modes; MNE; National Systems of Innovation.

JEL classification: F23; O30; O57.

\section{Introducción}

La investigación económica sobre inversión directa extranjera (IDE) se ha realizado fundamentalmente para los países más avanzados y ha sido escasa la evidencia empírica sobre formas de entrada en los países en desarrollo, pero el ascenso de estos últimos como receptores de flujos de IDE hace más necesario el análisis de los 
factores que intervienen en su capacidad de atracción. La emergencia de los países en desarrollo en la escena global se pone de manifiesto principalmente en la cuantía de IDE recibida. Por su parte, aunque el volumen de fusiones y adquisiciones (FyA) transfronterizas siga siendo inferior al de greenfield, los flujos de FyA han presentado incrementos mayores que los mostrados por las operaciones de nuevo emplazamiento sin socios locales, en forma de greenfield $^{1}$ (UNCTAD, 2005; 2007).

En este artículo estudiamos la relación entre las formas de entrada y los sistemas nacionales de innovación a través de un análisis multi-país, introduciendo explícitamente las diferencias según nivel de desarrollo de los países receptores ${ }^{2}$. Además de los aspectos que son recurrentes en la teoría económica -coste de los factores e imperfecciones del mercado-, otros elementos relacionados con el marco institucional y las capacidades de absorción de las economías actúan como factores determinantes de atracción de IDE. Estamos asumiendo, por tanto, el papel central que se le otorga a la tecnología y la innovación en la teoría de las empresas multinacionales (EMN), con el propósito de examinar la relevancia de los sistemas de innovación en la explicación de las formas de entrada.

Las divergencias internacionales en la distribución mundial de la IDE se deben tanto a factores de oferta como de demanda y las diversas estrategias de las EMN pueden tener efectos diferentes en el desarrollo económico de los países receptores (Ozawa, 1992; Lall, 2002; Meyer, 2004; Pearce, 2006; Rugman y Doh, 2008). Si bien la internacionalización de las EMN obedece a la combinación de ventajas de propiedad, de internalización y de localización, tal como se sugiere en la teoría OLI (Dunning, 2001), las estrategias de búsqueda de recursos, de mercados, de eficiencia y de conocimiento condicionan el potencial impacto de las EMN en el extranjero (Narula, 1996; Dunning, 2006). La obtención de recursos en el extranjero ha sido una estrategia predominante en la IDE dirigida hacia los países de menor desarrollo y la búsqueda de nuevos mercados ha estado más vinculada a las economías convergentes (Narula y Dunning, 2000). No obstante, hay dos novedades recientes que inciden en la relación entre IDE y países de acogida: por una parte, la creciente descentralización de operaciones de producción de las EMN a favor de los países en desarrollo y, por otra, la emergencia de FyA transfronterizas con objetivos de compra en estos países.

La investigación sobre formas de entrada ha estado centrada en el estudio de las ventajas de las empresas y de los factores que explicarían las decisiones empresariales, pero la evidencia empírica es menos abundante desde el punto de vista de las

\footnotetext{
${ }^{1}$ Aunque la información estadística de los flujos agregados de IDE incluye también otras formas de entrada -tales como licencias y joint-venture-, aquí abordamos las dos formas de internacionalización empresarial más relevantes: las greenfield y las FyA transfronterizas. Además, nótese que a lo largo del artículo denotaremos esos flujos agregados con el término "greenfield", haciendo así referencia explícita a la distinción con las fusiones y adquisiciones transfronterizas.

${ }^{2}$ En los últimos años, se ha revelado también una tendencia creciente de los flujos de salida de IDE procedentes de los países en desarrollo; no obstante, en este artículo se analiza exclusivamente la recepción de flujos.
} 
economías nacionales. En la literatura sobre internacionalización encontramos que los factores institucionales y culturales de los países receptores de IDE intervienen también en las decisiones empresariales sobre sus formas de entrada (Kogut y Singh, 1988; Rosenzweig y Singh, 1991; Harzing, 1999; Davis et al., 2000; Dunning, 2006). Las EMN pueden generar nuevas facilidades de producción, aportar nuevas prácticas de gestión y transferir nuevas tecnologías en el extranjero; no obstante, las subsidiarias pueden beneficiarse de los entornos locales ya que éstas también realizan la búsqueda de activos de conocimiento en las economías receptoras (Cantwell, 1989; 1995; Barkema y Vermeulen, 1998; Frost, 2001; Piscitello, 2004; McCann and Mudambi, 2005; Singh, 2007; Mudambi, 2008). La combinación de estos antecedentes en la literatura permite justificar que aquí se siga una aproximación integradora de los sistemas nacionales de innovación (SNI). El marco conceptual de los SNI se ha utilizado para realizar comparaciones internacionales de los estilos nacionales de gestión de la tecnología y la innovación (Freeman, 1987; Lundvall, 1992; Nelson, 1993; Mowery y Oxley, 1995; Cantwell y Molero, 2003), pudiendo aplicarse al análisis de las formas de entrada de IDE en relación con el desarrollo.

El vínculo entre las características de las economías receptoras y las formas de entrada de las EMN es complejo y puede entenderse a partir de la definición de una trayectoria de evolución conjunta: En una primera fase, las entradas de capital extranjero contribuyen a facilitar la industrialización de las economías en desarrollo, mientras que en fases posteriores a los primeros establecimientos, el cambio tecnológico y el marco institucional de las economías de acogida son elementos condicionantes de la recepción de nuevos flujos y también de la forma que adopten.

En este trabajo exploramos las formas de entrada de la IDE en la lógica de los países en desarrollo. En concreto, la hipótesis se construye a partir de la evolución de los países, que se define por su mayor capacidad de recepción de IDE y el tránsito hacia sistemas más atractivos para las FyA transfronterizas. Se trataría de contrastar la naturaleza no estática de los sistemas receptores, entendiendo que éstos se caracterizan por relaciones de producción verticales, por las relaciones entre empresas extranjeras y nacionales, y por las pautas de consolidación de las actividades nacionales de $\mathrm{I}+\mathrm{D}$ y de sus instituciones. El análisis cuantitativo se realiza para dos formas de entrada: IDE en forma de greenfield y de FyA, haciendo uso de información estadística para una muestra integrada por países desarrollados y economías en desarrollo durante un período de 7 años (1998-2004). Se recurre, por tanto, a un método de estimación de datos de panel dinámico y las fuentes de información estadística utilizadas han sido las bases de datos de la UNCTAD y del Banco Mundial.

El siguiente epígrafe está dedicado a los antecedentes teóricos. En el epígrafe 3, se desarrolla nuestra hipótesis de trabajo y, en el epígrafe 4, se realiza un análisis cluster y una descriptiva general de las variables por grupos de países. En el epígrafe 5 se propone el modelo empírico, tratando de revelar la importancia de los sistemas nacionales de innovación en la explicación de los flujos de IDE. En el epígrafe 6 se presentan algunas conclusiones y algunas ideas que pudieran servir para orientar la investigación futura. 


\section{Antecedentes en la literatura}

\subsection{Internacionalización empresarial y países receptores}

Las explicaciones que encontramos en la literatura económica y de los negocios internacionales sobre formas de entrada están fundamentalmente vinculadas a los objetivos empresariales de explotación de las ventajas competitivas y de reducción de los costes de transacción. El compromiso de recursos, la asunción de riesgos y las expectativas de aprendizaje en el extranjero son algunas de las fuerzas motrices que conducen a las empresas a internacionalizarse mediante la IDE (Agarwal y Ramaswami, 1992; Slangen y Hennart, 2007). Estos elementos, unidos a los motivos de las estrategias dominantes de las EMN, están en la base de la interacción entre IDE y nivel de desarrollo (Narula, 1996; Dunning, 2006).

La hipótesis de la senda de desarrollo de la inversión sostiene que los patrones de los flujos de entrada y de salida de IDE se relacionan con el nivel de desarrollo de los países (Dunning y Narula, 1996); de hecho, algunas economías de renta media no solo han demostrado ser muy atractivas para los inversores extranjeros sino que, además, también han comenzado a mostrar un activo comportamiento inversor a nivel internacional (Meyer, 2004; Cuervo-Cazurra, 2007; Santiso, 2008). Al tratar de contextualizar el problema, cabría distinguir, por tanto, entre aquellos países más atrasados -fundamentalmente en África y la mayoría de los que pertenecen al grupo de renta baja- y las economías emergentes que van ganando protagonismo en los mercados internacionales, entre los que se encontrarían las economías de Malasia, Indonesia y el grupo de los BRIC integrado por Brasil, Rusia, India y China. Tales diferencias refuerzan la necesidad de estudiar las formas de entrada y las posibilidades de la IDE en la difusión internacional de tecnología, atendiendo a las diferencias en el seno de las economías en desarrollo.

Desde la perspectiva empresarial, un aspecto clave es la elevada fragmentación de la cadena de valor que, en el plano internacional, condiciona los patrones de localización de las EMN, fundamentalmente en sectores intensivos en conocimiento en los que predomina la relocalización de las fases estandarizadas del proceso productivo en el extranjero e incluso la descentralización de actividades estratégicas, tales como la I+D (Mudambi, 2008). Los fundamentos microeconómicos de la descentralización de la $\mathrm{I}+\mathrm{D}$ hacia las empresas subsidiarias se sostienen en la existencia de flujos internacionales de conocimiento, desde el país originario de la EMN (casa matriz) hacia las unidades en el extranjero, y también a la inversa ${ }^{3}$. Igualmente relevante es el hecho de que las EMN han ido convirtiéndose en empresas multi-centro, capaces de explotar la diversidad de las localidades en las que están establecidas y de

${ }^{3}$ Esta propuesta se expone en el reciente modelo formal que explicita las implicaciones organizacionales que la descentralización de la I+D tiene en la integración de las empresas extranjeras en los sistemas productivos locales, reforzándose la posibilidad de spillovers de doble dirección (Sanna-Randaccio y Veugelers, 2007). 
generar un funcionamiento en red que implica formas específicas de gestión del conocimiento (Bartlett y Ghoshal, 1998; Mudambi, 2002). En la literatura sobre empresas subsidiarias se encuentran algunos argumentos a favor tanto de los flujos de conocimiento que tienen lugar en el seno de las organizaciones como de las posibilidades de aprovechamiento y explotación de las ventajas de red, permitiéndoles crear competencias enteramente nuevas y obtener un rendimiento óptimo de los activos de los países receptores; incluso tales argumentos han venido a poner de manifiesto la necesidad de reconsiderar la noción tradicional de ventaja competitiva (Rugman y Verbeke, 2001).

En cuanto a los efectos diversos que las EMN pueden generar en términos de desarrollo, hay que citar aquellos relacionados con la creación de empleo o de valor añadido en las economías receptoras que son consecuencia directa del inicio de la nueva actividad productiva. Además, pueden darse otros efectos que estarían relacionados con la creación y el comercio de activos intangibles así como con la posibilidad de generación de conocimiento y su difusión internacional (Cantwell, 1989; Ozawa, 1992). Estos últimos se aprecian en las diversas formas de transferencia tecnológica que se genera desde las empresas extranjeras hacia los sistemas productivos locales de acogida mediante, por ejemplo, la difusión de conocimiento incorporado en bienes y servicios y a través de la movilidad de trabajadores cualificados. También se definen por la competencia de las EMN, por los efectos de demostración de nuevas prácticas productivas, de nuevas técnicas y de controles de calidad en las relaciones verticales del sistema productivo (Rugman y Doh, 2008).

Con todo, cabe pensar que las consecuencias que tienen las formas de internacionalización en las economías receptoras dependen de las capacidades empresariales, tanto en el plano tecnológico como organizativo, de los proveedores de bienes y servicios intermedios y de los competidores locales. En el análisis de subsidiarias con mandatos específicos de creación o de explotación de competencias se contempla la existencia de diferentes intereses en las economías receptoras, subrayándose la importancia que tiene la búsqueda de activos de conocimiento (Cantwell y Mudambi, 2005). No obstante, la necesidad de activos, de tecnologías y de fuentes de información para la innovación en el entorno difiere según el sector industrial, ya que en las industrias intensivas en conocimiento es más acuciante el acceso a determinadas habilidades y experiencias, a universidades, a otras industrias y a agencias públicas (Chung y Alcacer, 2002).

\subsection{Formas de entrada y sistemas nacionales de innovación}

Al igual que las distintas estrategias, de búsqueda o de aumento de activos, implican relaciones diferenciadas con los sistemas nacionales de innovación, en las decisiones empresariales sobre formas de entrada también se tienen en cuenta las condiciones de los países de acogida, incluyendo las relacionadas con las empresas locales, con los factores de producción y otras a nivel de país. Aquí nos detendremos 
en las razones que explican por qué las empresas inversoras eligen FyA transfronterizas en lugar de misiones greenfield. Las FyA internacionales son más probables en industrias multipaís -en las que la competencia en cada país es independiente de la competencia en otros países en los que opera la EMN-porque las empresas extranjeras son más dependientes de los recursos locales y tienen mayor necesidad de ganar legitimidad en la economía de destino (Porter, 1986; Harzing, 1999). Al tiempo, las adquisiciones horizontales parecen estar generalmente dominadas por la búsqueda de mercados, de productos e incluso de marcas, y no tanto por la necesidad de disminuir costes de producción (Capron, 1999). En coherencia con la literatura, las FyA favorecen la integración vertical en los países receptores al aumentar la probabilidad de que las empresas subsidiarias tengan un mayor contenido local, ya que antes de la adquisición estas unidades eran empresas nacionales y, por tanto, estaban inmersas en el sistema productivo local (Belderbos et al., 2001).

La experiencia de las empresas en el mercado nacional constituye un aspecto crucial que facilita el aprendizaje de la cultura local y, por esta razón, las adquisiciones acaban siendo un modo más factible de obtener experiencia en el país receptor (Barkema y Vermeulen, 1998). La proximidad cultural entre el país emisor y receptor de la IDE junto a los menores niveles de incertidumbre, son factores que incrementan la probabilidad de entradas vía FyA (Kogut y Singh, 1988; Brouthers y Brouthers, 2000; 2003; Chang y Rosenzweig, 2001; Globerman y Shapiro, 2002; Shimizu et al., 2004). Aunque la literatura sobre negocios internacionales se haya centrado principalmente en los aspectos legales y culturales (Scott, 1981; 1983), el conjunto de elementos que integran el entorno local de las empresas subsidiarias es más amplio, incluyendo la estructura industrial, las capacidades tecnológicas y la regulación gubernamental (Rosenzweig y Singh, 1991; Davis et al., 2000). Además, se ha demostrado que el grado de madurez de estos elementos del entorno pueden ofrecer nuevas oportunidades de inversión a las EMN incluso en países culturalmente distantes y en economías emergentes (Hitt et al., 2006).

Hay también evidencia empírica sobre FyA que confirma una mayor probabilidad de esta forma de entrada cuando el ámbito geográfico de actuación de las empresas es más amplio y cuando las EMN tienen una mayor experiencia internacional (Mudambi y Mudambi, 2002). También es más probable que empresas más diversas y con menor intensidad de $\mathrm{I}+\mathrm{D}$ sean las que acudan a comprar capacidades tecnológicas en otros países mediante FyA y que la propensión a adoptar esta forma de entrada aumente cuando las empresas locales hayan desarrollado sus sistemas de distribución y tengan un mayor conocimiento del mercado local (Harzing, 1999). La cuestión es que la forma de entrada puede tener efectos en la transferencia de conocimiento, aspecto que se confirma en el análisis de Yang et al. (2008) acerca de los determinantes de los flujos de conocimiento bidireccionales en los países en transición del Centro y Este de Europa, detectándose la existencia de diferencias nacionales (efecto-país) significativas.

Por todo ello, la aproximación de los sistemas nacionales de innovación permite estudiar las relaciones entre formas de entrada y las características de los países de 
acogida de la IDE a nivel agregado. Este marco conceptual hace referencia a la influencia y evolución conjunta de las actividades de producción y de las instituciones en la generación de conocimiento ${ }^{4}$. En un sentido amplio, en este modelo analítico se combinan los aspectos más tradicionales de las relaciones verticales de los sistemas productivos -incluyendo el aprendizaje por la práctica y el aprendizaje por la búsqueda- con algunos fundamentos microeconómicos basados en el modelo interactivo de Kline y Rosenberg (1986), las fuentes del conocimiento para la innovación (Von Hippel, 1988) y la dimensión institucional que promueve el aprendizaje interactivo (Lundvall et al., 2002).

Esta perspectiva es igualmente válida para su aplicación al análisis de los países en desarrollo si se adopta la definición amplia de sistema, tal como fue presentada por sus precursores (Freeman, 1987; Lundvall, 1992). En cualquier sistema, el cambio tecnológico y el crecimiento económico son el resultado de la innovación que realiza todo el universo de empresas, reflejándose el desempeño del sistema en el cambio tecnológico y organizativo de los sistemas industriales como un todo (Lundvall et al., 2009). El funcionamiento de las universidades y centros de investigación, el sistema de patentes y de publicaciones, la política tecnológica y los programas de investigación, son todos ellos elementos del sistema de innovación. En su definición amplia también se incorporan el sistema educativo y financiero, el entorno competitivo y las políticas laborales, así como otras organizaciones e instituciones, incluyéndose además la calidad de las relaciones entre clientes y productores, el grado de confianza en la sociedad, la distribución de la renta, la transparencia, la calidad regulatoria, las políticas de restricción de la corrupción y los conflictos étnicos.

El enfoque de sistemas nacionales de innovación resulta pues apropiado en el análisis de las formas de entrada de la IDE ya que es revelador de las diferencias de los sistemas productivos y de investigación a nivel de país. Permite además insertar las capacidades de absorción y de aprendizaje de los individuos y organizaciones como elementos determinantes de los procesos de concentración de la actividad económica, lo que es consistente con la probada evidencia empírica acerca de los clusters tecnológicos y su capacidad para crear un entorno de conocimiento especializado que hace a los territorios más atractivos para la recepción de EMN (Mudambi, 2002; Tihanyi and Roath, 2002; Cantwell and Molero, 2003). De la revisión anterior puede extraerse que las empresas, al elegir entrar en un entorno extranjero por la vía de las FyA, estudian las ventajas de acceder a las redes de distribución y de conocimiento locales, a las relaciones institucionales y a la cultura local. En este sentido, las características institucionales de las economías de acogida resultan ser determinantes de la transferencia de tecnología, mientras que el desarrollo socioeconómico gana importancia en la toma de decisiones empresariales y condiciona, además, su forma de entrada.

\footnotetext{
${ }^{4}$ Este marco conceptual considera instituciones informales (la confianza) e instituciones formales (derechos de propiedad intelectual o la regulación contractual).
} 


\section{Desarrollo de hipótesis}

En este trabajo abordamos la relación entre las formas de entrada de la IDE y el nivel de desarrollo de los países receptores para constrastar si los factores del sistema nacional de innovación de éstos condicionan el tránsito hacia entradas vía FyA. Esta forma de entrada, frente a la greenfield, implica una mayor y más rápida interacción con las capacidades del entorno y, por tanto, podría ser más dependiente del avance de los países. Las FyA ocurren, literalmente, cuando existen objetivos de compra, un aspecto que está condicionado por las capacidades empresariales de los sistemas nacionales de innovación locales, así como por otros activos relacionados con la disponibilidad de conocimiento especializado y de capacidades científicas y tecnológicas. El objetivo de nuestro trabajo empírico será valorar esa relación a través de dos componentes: El primero se refiere a los flujos de llegada de IDE - greenfield-que no discriminan entre los aspectos más cualitativos de la inversión y que, generalmente, requieren la generación de capacidades específicas en las economías de acogida (Agarwal y Ramaswami, 1992; Slangen y Hennart, 2007). El segundo está relacionado con un mayor grado de implicación de los flujos de capital extranjero en las economías receptoras y se aproxima por el volumen anual de FyA transfronterizas, asumiéndose que esta forma de entrada genera un mayor grado de interacción con las economías locales (Xu, 2000).

Las inversiones greenfield usualmente requieren el desarrollo de relaciones con los agentes locales para acceder al abastecimiento de consumos intermedios, el establecimiento de canales de distribución e incluso la búsqueda de socios tecnológicos. La mayor ventaja de las FyA es que estas operaciones permiten el acceso directo a las relaciones verticales de las empresas objetivo de la adquisición. De hecho, las subsidiarias adquiridas tienen un contenido local mayor que también afecta a los activos de conocimiento, como ha sido demostrado por evidencia empírica previa (Belderbos et al., 2001, Marín y Álvarez, 2009). Entre los resultados del análisis de empresas japonesas que se lleva a cabo en Belderbos (2003), cabe señalar que hay notables diferencias en la intensidad de $\mathrm{I}+\mathrm{D}$ de las subsidiarias manufactureras extranjeras y que tales diferencias dependen de las formas de entrada, siendo sustancial y significativamente más alta la intensidad de I+D de las empresas adquiridas. Al tiempo, la evidencia empírica sobre la internacionalización de las EMN suecas también muestra que es más probable que las subsidiarias adquiridas realicen $\mathrm{I}+\mathrm{D}$ y que las FyA han estado más claramente motivadas por estrategias de búsqueda de activos de conocimiento que las operaciones greenfield (Bertrand et al., 2007).

Una idea que resulta clave es que los países avanzados muestran mejores capacidades tecnológicas y empresariales, y que ello puede constituir un importante determinante en la atracción de FyA. De hecho, aunque las FyA transfronterizas que han tenido como destino los países en desarrollo hayan representado una moderada proporción de los flujos de IDE, las empresas de estos países están viéndose envueltas de forma creciente en FyA (UNCTAD, 2005). Lo que proponemos es, por tanto, demostrar si existe una relación potencial entre las distintas formas de entrada y los 
sistemas nacionales de innovación de estos países. Esa relación puede describirse a partir de un proceso dinámico que se caracteriza por la evolución conjunta de la IDE y los sistemas receptores, de la siguiente forma: En una primera fase de desarrollo, la IDE es menos diversa y menos dependiente de las capacidades de absorción y el marco institucional de los países receptores; en fases posteriores y más avanzadas de desarrollo, aumenta la capacidad de atracción de otras formas de IDE, siendo mayor también la probabilidad de recibir FyA. Al hilo de este proceso cabe aproximar las posibilidades de las economías para incorporarse a los cambios de tendencia de la IDE a nivel mundial, en la que las FyA ganan protagonismo, y confirmar que se trata de un proceso escasamente determinista; los elementos cualitativos de los SNI contribuirían a explicar el tránsito desde las operaciones greenfield a una mayor presencia de FyA.

En particular, hay dos aspectos especialmente relevantes al adoptar una concepción dinámica del problema. En primer lugar, el hecho de que las estrategias empresariales van cambiando con el tiempo y que las empresas extranjeras acaban por integrarse más en los sistemas locales (Pearce, 1999). En este sentido, las empresas operando en contextos foráneos no solo pueden contribuir al desarrollo del sistema productivo local sino que suponen incentivos a la llegada de nueva IDE a esa localidad ya que las EMN prefieren llevar a cabo inversiones en aquellos países en los que ya son activas; su experiencia pasada contribuye a definir, por tanto, el proceso acumulativo de las entradas de IDE (Davidson, 1980; Mudambi, 1995). En segundo lugar, la importancia de las inversiones en I+D como factor determinante de desarrollo que incrementa las capacidades de absorción de conocimiento en los sistemas de innovación receptores. El concepto de capacidades de absorción, adoptado de la noción micro que fuera formulada por Cohen y Levinthal (1990), ha de entenderse como las posibilidades de las organizaciones para beneficiarse de las innovaciones llevadas a cabo externamente, por otras empresas, y que constituyen una segunda fase del aprendizaje.

De acuerdo a la teoría y a la evidencia empírica disponible, trataremos de comprobar si la entrada de IDE y de FyA transfronterizas varían con la importancia relativa de las características de los sistemas nacionales de innovación receptores. Cabe esperar que un SNI más avanzado presente una mayor probabilidad de atraer flujos de entrada y, particularmente, en forma de FyA. Para hacer operativa esta hipótesis, la primera pregunta a responder es si la presencia previa de capital extranjero afecta positivamente la forma de entrada y si su efecto es más importante en el caso de las FyA. Cabe plantearse también si las capacidades de absorción afectan positivamente la recepción de flujos y si su efecto será más notable en las FyA. Finalmente, el sistema de innovación de un país avanzado y tecnológicamente activo suele caracterizarse por una mayor estabilidad institucional; por ello, puede comprobarse si marcos institucionales más estables tienen un efecto positivo sobre las diferentes formas de entrada y si ese efecto es más considerable en las FyA. Al mismo tiempo, será necesario incluir algunos factores económicos que determinan las formas de entrada, tales como las características de los mercados locales -tamaño y dinamismo-, la 
integración de los países en los mercados internacionales -grado de apertura- y las condiciones laborales - costes y cualificaciones de la mano de obra. Cada uno de estos factores estará representado por su correspondiente indicador, haciéndose operativo en el contraste de la hipótesis a través de las respectivas variables independientes; se procederá a una descripción más detallada tanto de las variables como de su medición en el epígrafe 5.

\section{Análisis descriptivo de los grupos de países}

El stock de IDE y las capacidades de absorción son dos aspectos que, junto a la estabilidad institucional, condicionan los sistemas nacionales receptores de IDE. Las capacidades de absorción pueden medirse, en el plano agregado, a través de los gastos nacionales en I+D (Narula et al., 2002) y aunque puede reclamarse que sea una medida imperfecta de tales capacidades, hay que tener en cuenta que este indicador viene a revelar no solo las actividades de generación de conocimiento sino también los esfuerzos de las organizaciones para asimilar nuevo conocimiento ${ }^{5}$. El stock de IDE aproxima, por su lado, el carácter acumulativo de la inversión así como la interacción de las EMN con los sistemas locales. Las posibilidades de desarrollo de los países vienen condicionadas por su esfuerzo en la realización de actividades conducentes a la generación de conocimiento. Es precisamente en ese ámbito donde las diferencias entre economías de disímil nivel de renta se hacen especialmente presentes, aceptándose que la trayectoria de crecimiento económico está determinada por el avance tecnológico de los países.

De acuerdo a nuestra hipótesis, cabe esperar que a medida que se desarrolla el SNI de los países receptores de IDE, se dé un cambio en las estrategias de internacionalización de las EMN, siendo más probable que se produzcan nuevas inversiones vía FyA. Para contrastarla, se trabaja con una muestra de países que incluye países desarrollados y en desarrollo. Al considerar la clasificación del Banco Mundial que agrupa los países en función de su renta per cápita, se distingue entre países de renta alta, países de renta baja y un amplio grupo de países de renta media. Este último se divide, a su vez, en economías de renta media-alta y media-baja, siendo notable la heterogeneidad que revelan las capacidades tecnológicas en el seno de estos dos grupos de países (Álvarez y Magaña, 2007). Nótese que en el grupo de renta media se integran las economías emergentes, que no sólo han experimentado un rápido crecimiento económico sino que han llegando a ser muy atractivas para la inversión extranjera (Hitt et al., 2006) y que están mostrando un importante potencial para desarrollar tecnologías, por ejemplo, en Brasil, China e India, gracias a la acción combinada del estado, el capital extranjero y el capital nacional.

${ }^{5}$ Sería más apropiado aproximar estas capacidades a partir de la consideración conjunta de otros indicadores tecnológicos, tales como las patentes. Sin embargo, la falta de disponibilidad de información estadística para la muestra de países aquí utilizada impide incluir otras variables en el análisis. 
El esfuerzo en $\mathrm{I}+\mathrm{D}$ de los grupos de países según nivel de renta, ilustrado en el Gráfico 1, nos permite comprobar que es amplia la distancia existente entre el gasto en I+D -como proporción del PIB- de los países de renta alta y los dos grupos de renta media ${ }^{6}$. El nivel promedio de esfuerzo en $\mathrm{I}+\mathrm{D}$ de los países de mayor renta ha llegado a alcanzar un valor de 2,40\% en 2005. Por su parte, en el grupo de renta media, encontramos que el esfuerzo en I+D de los países de renta media-baja ha llegado a ser el 1,00\% en 2005, mientras que el grupo de renta media-alta muestra unos valores inferiores en los dos años considerados. El grupo de países de renta mediabaja ha experimentado, de hecho, el mayor crecimiento acumulado de los gastos en $\mathrm{I}+\mathrm{D}$ entre 1998 y 2005 (incremento cercano a 6\%) lo que revela que haya reducido la distancia que le separa de los países más desarrollados en el esfuerzo en I+D.

\section{GRÁFICO 1}

GASTO EN I+D COMO PORCENTAJE DEL PIB

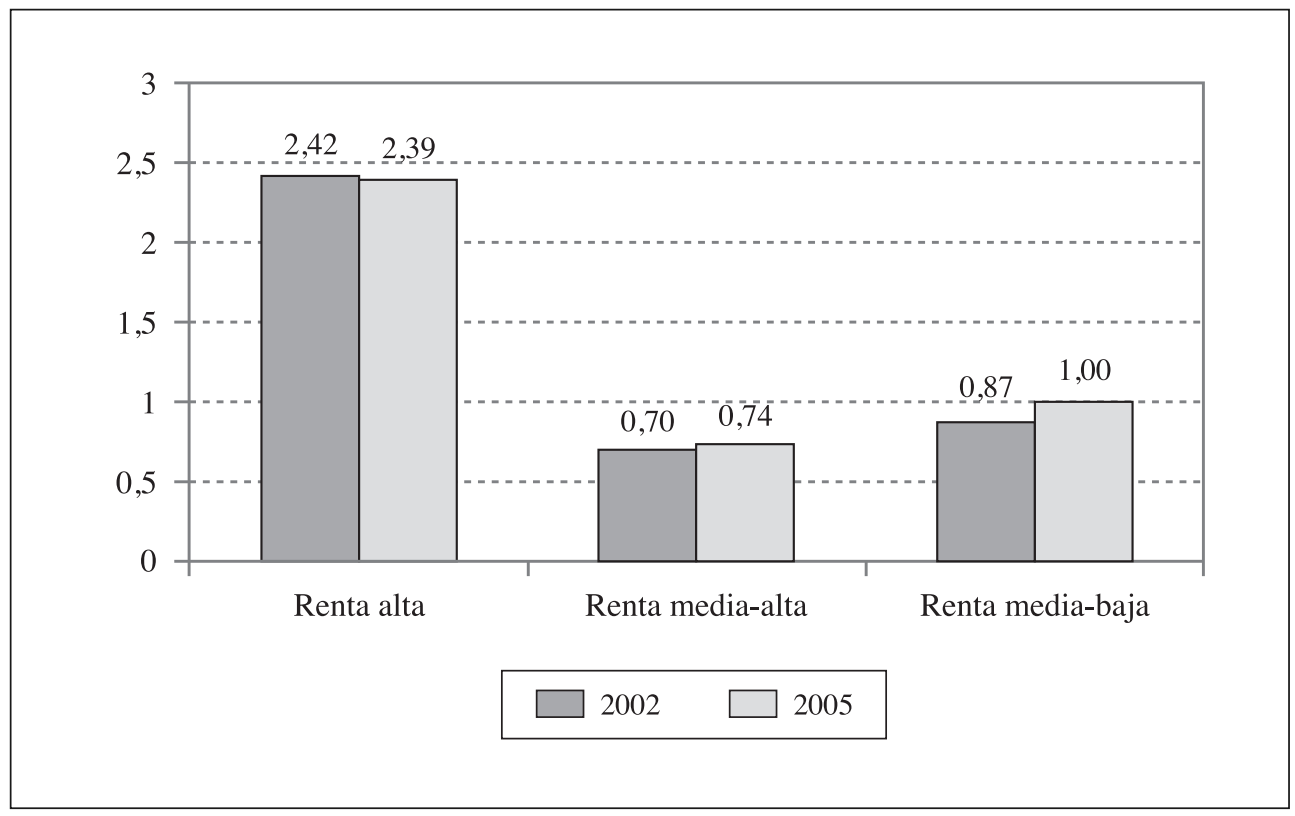

Nota: En el grupo de renta media-alta, los gastos en I+D de 1998 corresponden a 1999.

Fuente: elaboración propia con datos de Banco Mundial: World Development Indicators.

Estos resultados vienen a justificar una nueva agrupación de los países en la que explícitamente se tengan en cuenta sus capacidades de absorción. Por ello, se ha ela-

${ }^{6}$ El criterio aquí utilizado para agrupar a los países ha sido el de renta per capita que utiliza el Banco Mundial. Puede percibirse que está ausente el grupo de países de renta baja, que ha sido deliberadamente eliminado por la escasa disponibilidad de información estadística disponible. Los valores que se ilustran en el gráfico corresponden a la media ponderada de cada uno de los grupos de renta. 
borado un análisis cluster con el fin de identificar los diferentes grupos de países en función de las características de los SNI. El análisis cluster es una técnica de análisis multivariante que se emplea para establecer diferentes grupos de individuos sobre la base de características comunes (Aldenderfer y Blashfield, 1984; Bailey, 1994; Ketchen y Shook, 1996). Como los resultados de un análisis cluster pueden diferir en función del método aplicado, se ha optado por llevar a cabo uno de tipo jerárquico (considerando el método de Ward y las distancias euclídeas al cuadrado) y otro de tipo iterativo (método de K-medias) con el fin de comprobar la robustez de los resultados obtenidos. Las variables incluidas en el análisis han sido el gasto en I+D (medido como porcentaje del PIB) y el stock de IDE, ya que ambas condicionan los sistemas nacionales de innovación.

El resultado del análisis cluster lleva a una agrupación de los países incluidos en la muestra que es algo diferente a la que resulta haciendo uso del criterio de renta per capita. En el Cuadro 1 puede observarse la configuración de los cluster obtenidos: El cluster 1 lo forman, por lo general, países de renta media que cuentan con "sistemas menos avanzados", grupo al que se incorporan dos economías de renta alta -Kuwait e Islandia- con una importante especialización en recursos naturales. El cluster 2 está formado por países con sistemas en una fase "de avance intermedio"; a este grupo pertenecen también las economías europeas de Grecia, Hungría y República Checa, además de Nueva Zelanda. Por último, el cluster 3 integra a los "sistemas más avanzados", pudiendo observarse que junto a la mayoría de las economías de renta alta se integran México, Brasil y China, países que destacan entre los países de renta media tanto por el stock de IDE como por el esfuerzo en I+D (Álvarez y Magaña, 2007).

También se han calculado algunos estadísticos descriptivos que contribuyen a caracterizar de forma sencilla los grupos de países que se generaron en el análisis cluster. Tales estadísticos, en el Cuadro 2, se refieren a cada una de las formas de entrada (IDE y FyA), a las características de los sistemas de innovación de los países receptores (capacidades de absorción y stock de IDE) así como a los factores económicos que condicionan la entrada de IDE. Estos últimos incluyen el tamaño y dinamismo del mercado (aproximados por el PIB y el crecimiento del PIB, respectivamente), aspectos del mercado laboral (nivel de salarios y de capital humano medido mediante la cualificación de la población - matriculados en educación secundaria respecto a la población total en edad de cursar secundaria), y el grado de apertura de los países (aproximado por el peso que representan los flujos de comercio exterior en el PIB). Respecto a los SNI, también se incluye una variable de marco institucional; aun siendo una medida imperfecta, la estabilidad institucional se aproxima mediante el Indicador de Gobernabilidad Governance Matters Indicator ${ }^{7}$ (GMI) -véase Kaufmann et al. (2009).

\footnotetext{
${ }^{7}$ Este indicador es el resultado del promedio de seis indicadores diferentes referentes a la voz y rendición de cuentas, la estabilidad política y ausencia de violencia, la efectividad gubernamental, la calidad regulatoria, el Estado de derecho y el control de la corrupción. Para cada uno de ellos y para cada país, se han recopilado 352 indicadores procedentes de diversas fuentes como organismos internacionales, agencias de rating y otras -véase Kaufmann et al. (2009).
} 


\section{CUADRO 1}

RESULTADOS DEL ANÁLISIS CLUSTER

\begin{tabular}{|c|c|c|c|c|c|}
\hline $\begin{array}{c}\text { Cluster } 1 \\
\text { Sistemas } \\
\text { menos } \\
\text { avanzados }\end{array}$ & Distancia & $\begin{array}{c}\text { Cluster } 2 \\
\text { Sistemas } \\
\text { de avance } \\
\text { intermedio }\end{array}$ & Distancia & $\begin{array}{c}\text { Cluster } 3 \\
\text { Sistemas } \\
\text { más } \\
\text { avanzados }\end{array}$ & Distancia \\
\hline Letonia & 0,198 & Turquía & 0,457 & Singapur & 0,160 \\
\hline Costa Rica & 0,395 & Grecia & 0,486 & Australia & 0,178 \\
\hline Urugüay & 0,403 & Túnez & 0,518 & Irlanda & 0,414 \\
\hline Bulgaria & 0,596 & Venezuela & 0,602 & Suiza & 0,436 \\
\hline Chipre & 0,635 & Marruecos & 0,670 & Italia & 0,491 \\
\hline Lituania & 0,656 & India & 0,725 & Canadá & 0,579 \\
\hline Bielarrusia & 0,730 & Hungría & 0,746 & Brasil & 0,611 \\
\hline Malta & 0,834 & Egipto & 0,748 & Dinamarca & 0,630 \\
\hline Georgia & 0,872 & Polonia & 0,805 & España & 0,796 \\
\hline Estonia & 0,886 & Tailandia & 0,809 & China & 0,808 \\
\hline Bolivia & 0,893 & Malasia & 0,812 & Suecia & 0,837 \\
\hline Aerbayán & 0,914 & Colombia & 0,878 & Alemania & 0,940 \\
\hline Macedonia & 0,970 & Chile & 0,887 & Japón & 0,947 \\
\hline Eslovaquia & 1,091 & Kazakhstan & 0,891 & Bélgica & 0,948 \\
\hline Armenia & 1,176 & Portugal & 0,907 & Luxemburgo & 0,989 \\
\hline Mauricio & 1,263 & Rumarían & 0,919 & Francia & 1,055 \\
\hline El Salvador & 1,314 & Argentina & 1,008 & Noruega & 1,225 \\
\hline Croacia & 1,373 & Rusia & 1,018 & Austria & 1,226 \\
\hline Ucrania & 1,453 & Panamá & 1,031 & Corea-Sur & 1,242 \\
\hline Eslovenia & 1,483 & Rep. Checa & 1,139 & Reino Unido & 1,377 \\
\hline Kuwait & 1,538 & Nueva Zelanda & 1,165 & México & 1,464 \\
\hline Paragüay & 1,585 & Perú & 1,495 & Finlandia & 1,579 \\
\hline Honduras & 2,032 & Trinidad Tobago & 1,556 & Hong-Kong & 1,617 \\
\hline \multirow[t]{2}{*}{ Islandia } & 2,205 & Ecuador & 2,015 & Israel & 1,979 \\
\hline & & & & Estados Unidos & 2,320 \\
\hline
\end{tabular}

Fuente: elaboración propia. 
Los resultados, en el Cuadro 2, nos permiten describir de forma sencilla los grupos de países que se generaron en el análisis cluster previamente comentado. Puede comprobarse que los valores promedio más elevados en las dos formas de entrada de IDE corresponden a los países del cluster 3 (sistemas más avanzados) aunque también la dispersión de estas dos variables es más alta en este grupo -todas las especificaciones de las variables se encuentran en el Cuadro A1 del Anexo. Las diferencias que muestra este grupo de países con SNI más avanzados respecto a los otros dos conglomerados también son notables en el stock de IDE, en el indicador institucional (GMI) y en el esfuerzo en I+D, siendo más elevada la dispersión de estas dos últimas variables en el cluster 1 (sistemas menos avanzados).

CUADRO 2

CARACTERÍSTICAS DE LOS CLUSTERS DE PAÍSES, 1998-2004

\begin{tabular}{|c|c|c|c|c|c|c|}
\hline & \multicolumn{2}{|c|}{$\begin{array}{l}\text { SNI menos avanzados } \\
\text { Cluster } 1\end{array}$} & \multicolumn{2}{|c|}{$\begin{array}{l}\text { SNI avance intermedio } \\
\text { Cluster } 2\end{array}$} & \multicolumn{2}{|c|}{$\begin{array}{l}\text { SNI más avanzados } \\
\text { Cluster } 3\end{array}$} \\
\hline & Media & $\begin{array}{l}\text { Desv. Típ/ } \\
\text { Media }\end{array}$ & Media & $\begin{array}{l}\text { Desv. Típ/ } \\
\text { Media }\end{array}$ & Media & $\begin{array}{l}\text { Desv. Típ/ } \\
\text { Media }\end{array}$ \\
\hline $\begin{array}{l}\text { IDE (millones de dólares } \\
\text { EEUU constantes, 2000) }\end{array}$ & 564,29 & 1,16 & $2.763,64$ & 1,04 & $26.421,51$ & 1,66 \\
\hline $\begin{array}{l}\text { FyA (millones de dólares } \\
\text { EEUU constantes, 2000) }\end{array}$ & 256,36 & 1,93 & $1.379,97$ & 1,66 & $21.533,97$ & 2,18 \\
\hline $\begin{array}{l}\text { Stock de IDE (millones } \\
\text { de dólares EEUU constantes, } \\
\text { 2000) }\end{array}$ & $2.964,08$ & 0,91 & $25.957,10$ & 0,67 & $211.788,95$ & 1,28 \\
\hline I+D/PIB (porcentaje) & 0,56 & 1,02 & 0,49 & 0,66 & 2,12 & 0,47 \\
\hline $\begin{array}{l}\text { Estabilidad institucional } \\
\text { (Indicador de Gobernabilidad) }\end{array}$ & 0,15 & 4,93 & 0,19 & 3,48 & 1,34 & 0,35 \\
\hline $\begin{array}{l}\text { PIB (millones de dólares } \\
\text { EEUU constantes, 2000) }\end{array}$ & $12.530,08$ & 0,74 & $83.805,89$ & 0,79 & $1.090 .633,59$ & 1,95 \\
\hline Crecimiento del PIB (\%) & 4,23 & 0,84 & 3,33 & 1,26 & 2,96 & 0,87 \\
\hline Apertura comercial (\%) & 99,39 & 0,34 & 80,83 & 0,54 & 93,66 & 0,75 \\
\hline $\begin{array}{l}\text { Coste laboral (sueldos } \\
\text { y salarios, millones de dólares } \\
\text { EEUU constantes, 2000) }\end{array}$ & $2.384,99$ & 1,12 & $9.798,69$ & 0,73 & $39.917,22$ & 1,56 \\
\hline $\begin{array}{l}\text { Capital humano (matriculados } \\
\text { en secundaria) }\end{array}$ & 86,02 & 0,18 & 82,35 & 0,22 & 108,90 & 0,26 \\
\hline
\end{tabular}

Fuente: elaboración propia. 
Igualmente significativas son las diferencias entre los grupos de países en cuanto al nivel de salarios, al tamaño de la economía y al capital humano. Sin embargo, los países pertenecientes al cluster 1 son los que muestran un mayor dinamismo del mercado interior y una mayor apertura a los mercados internacionales, con valores superiores a los obtenidos en el cluster 2 (sistemas de avance intermedio), siendo la dispersión de estas variables más baja en el cluster 1.

\section{Análisis Empírico}

\subsection{El modelo empírico}

El modelo empírico que aquí desarrollamos trata de explicar las formas de entrada (flujos greenfield y de FyA transfronterizas) en función de algunas características de los SNI de los países receptores: el carácter acumulativo del capital extranjero (IDEStock), la intensidad en I+D (ID) y el marco institucional (GMI). También se incluyen aquellos aspectos económicos más convencionales en la atracción de IDE, tales como el tamaño y el crecimiento del mercado interno (PIB y $\triangle \mathrm{PIB}$ ), el grado de apertura (AP), los costes laborales (W) y el nivel de capital humano $(\mathrm{CH})$. Todas las variables anteriormente mencionadas se incluyen en el modelo en logaritmos, con la excepción de GMI por ser éste un indicador. En la estimación se considera como variable dependiente, en primer lugar, la inversión greenfield (IDE) y, en una segunda estimación, las FyA transfronterizas (FyA). El modelo viene representado en la ecuación (1), incluyéndose variables ficticias de tiempo y de país para poder controlar por aquellos impactos macroeconómicos no explicitados en el modelo:

$$
\begin{aligned}
y_{i t}= & \alpha_{1} I D E S t o c k_{i t}+\alpha_{2} I D_{i t}+\alpha_{3} G M I_{i t}+\alpha_{4} P I B_{i t}+\alpha_{5} \Delta P I B_{i t}+ \\
& +\alpha_{6} A P_{i t}+\alpha_{7} W_{i t}+\alpha_{8} C H_{i t}+\eta_{d i}+v_{d t}+\varepsilon_{i t}
\end{aligned}
$$

El método de estimación, la disponibilidad de datos de panel y la dimensión temporal son aspectos cruciales en el contraste de la hipótesis referente al papel que las características de los SNI juegan en la explicación de la evolución de los flujos de IDE. En consecuencia, el modelo anteriormente especificado se estima siguiendo una aproximación dinámica debido a la estructura inherentemente endógena del mismo. Es decir, la variable dependiente y sus retardos pueden estar correlacionados con las variables independientes, de manera que los valores pasados de los diferentes determinantes de la inversión pueden condicionar la forma de entrada en el momento actual. El método generalizado de los momentos (GMM) transforma el modelo tomando primeras diferencias para eliminar los efectos individuales no observados y emplea todos los posibles retardos de los regresores como instrumentos para eliminar las posibles correlaciones con el efecto individual (Arellano y Bond, 1991). En 
una extensión del estimador GMM, denominada system-GMM, se tienen en cuenta tanto los instrumentos originales en niveles para las ecuaciones en primeras diferencias, como los instrumentos en primeras diferencias para las ecuaciones en niveles (Arellano y Bover, 1995; Blundell y Bond, 1998). En este procedimiento de estimación, las variables predeterminadas en niveles se instrumentan mediante los retardos de sus propias diferencias; éste es el que se ha adoptado en la estimación debido a sus mejores resultados y a sus ventajas sobre el estimador de primeras diferencias del GMM, ya que explota todos los momentos disponibles.

\subsection{Resultados y discusión}

Los resultados de la estimación permiten confirmar que la entrada vía greenfield presenta una relación positiva con la presencia previa de capital extranjero en la economía receptora. El tamaño y el dinamismo del mercado interior son también elementos importantes en la atracción de este tipo de inversión, mientras que los costes laborales actúan negativamente y el grado de apertura y el nivel de capital humano no presentan un efecto significativo (columna 1, Cuadro 3). Por otra parte, las capacidades de absorción y el marco institucional no parecen tener gran capacidad explicativa en la atracción de inversión greenfield. Cuando se controla por los grupos de países obtenidos en el análisis cluster (columna 2, Cuadro 3), los resultados obtenidos permanecen inalterados, no pareciendo existir diferencias entre los países con SNI más avanzados, de avance intermedio y menos avanzados.

En cuanto a las FyA transfronterizas, la presencia previa de IDE también se muestra relevante en esta forma de entrada, siendo su efecto superior que en el caso de inversiones greenfield. No obstante, las capacidades de absorción y la estabilidad institucional ganan protagonismo al ser significativas y estar relacionadas positivamente con la entrada de inversión vía FyA (columna 3, Cuadro 3). Sin embargo, ni el tamaño del mercado interno ni su dinamismo, ni tampoco el capital humano, parecen desempeñar un papel importante en la atracción de FyA transfronterizas. Cuando se controla por los diferentes conglomerados (columna 4, Cuadro 3), los resultados revelan que el esfuerzo en I+D refuerza el argumento planteado en este trabajo con respecto al papel de los SNI en la explicación de los flujos de IDE que tienen un mayor nivel de compromiso e interacción con el país de destino. Este efecto se aprecia en el signo positivo y significativo que adopta el coeficiente de la interacción de la variable de esfuerzo en I+D y los países de SNI menos avanzados y de avance intermedio, respectivamente. 


\section{CUADRO 3}

RESULTADOS DE LA ESTIMACIÓN

\begin{tabular}{|l|c|c|c|c|}
\hline \multirow{2}{*}{} & \multicolumn{2}{|c|}{ IDE } & \multicolumn{2}{c|}{ FyA } \\
\cline { 2 - 5 } & $(1)$ & $(2)$ & $(3)$ & $(4)$ \\
\hline IDEStock & $0.859^{* * *}$ & $0.851^{* * *}$ & $0.897^{* * *}$ & $0.797^{* * *}$ \\
& $(0.057)$ & $(0.065)$ & $(0.134)$ & $(0.138)$ \\
\hline ID & 0.1624 & & $0.621^{* *}$ & \\
& $(0.105)$ & & $(0.249)$ & \\
\hline GMI & 0.110 & 0.111 & $0.599^{* *}$ & $0.617^{* *}$ \\
& $(0.115)$ & $(0.108)$ & $(0.278)$ & $(0.275)$ \\
\hline PIB & $0.268^{* *}$ & $0.293^{* *}$ & 0.102 & 0.238 \\
& $(0.119)$ & $(0.124)$ & $(0.245)$ & $(0.217)$ \\
\hline SPIB & $0.778^{* *}$ & $0.713^{* *}$ & 1.278 & 0.893 \\
& $(0.355)$ & $(0.347)$ & $(1.253)$ & $(0.968)$ \\
\hline AP & -0.168 & -0.171 & $-1.055^{* * *}$ & $-1.085^{* * *}$ \\
& $(0.167)$ & $(0.177)$ & $(0.343)$ & $(0.312)$ \\
\hline W & $-0.278^{* *}$ & $-0.305^{* *}$ & -0.057 & -0.194 \\
& $(0.139)$ & $(0.139)$ & $(0.267)$ & $(0.241)$ \\
\hline CK & -0.171 & -0.153 & 0.059 & 0.257 \\
& $(0.292)$ & $(0.311)$ & $(0.508)$ & $(0.552)$ \\
\hline ID*Cluster 1 & & 0.247 & & $1.245^{* * *}$ \\
(SNI menos avanzados) & & $(0.212)$ & & $(0.356)$ \\
\hline ID*Cluster2 & & 0.182 & & $0.494^{*}$ \\
(SNI avance intermedio) & & $(0.122)$ & & $(0.265)$ \\
\hline ID* Cluster3 & & 0.086 & & 0.073 \\
(SNI más avanzados) & & $(0.156)$ & & $(0.249)$ \\
\hline Test de Hansen Chi^2 & 35.77 & 35.76 & 39.92 & 40.87 \\
\hline Test de Arellano-Bond: AR(1) & $-2.67^{* * *}$ & $-2.68^{* * *}$ & $-1.92^{* *}$ & $-1.94^{* *}$ \\
\hline Test de Arellano-Bond: AR(2) & -0.60 & -0.62 & -0.67 & -0.72 \\
\hline Número de observaciones & 404 & 404 & 364 & 364 \\
\hline Número de individuos & 73 & 73 & 71 & 71 \\
\hline & & & & \\
\hline & & & & \\
\hline
\end{tabular}

Errores estándares robustos entre paréntesis: * significatividad al 10\%; $\quad * *$ significatividad al 5\%; $\quad * * *$ significatividad al $1 \%$.

Fuente: elaboración propia. 
Estos resultados distinguen claramente el comportamiento de las dos formas de entrada, aunque las dos tienen en común el papel significativo de la presencia de capital extranjero en las economías. Por lo tanto, se confirma la importancia de los elementos cualitativos relacionados con los SNI en el tránsito desde aquellas formas más tradicionales de IDE a la entrada vía FyA. Los resultados sugieren que las capacidades de absorción son más importantes como determinantes en la explicación de las FyA transfronterizas en aquellos países que se encuentran en una fase de desarrollo de sus sistemas productivos y tecnológicos.

El hecho es que, a pesar de la dificultad que supone integrar diferentes culturas empresariales, en la internacionalización vía FyA las empresas inversoras están más interesadas en acceder a las relaciones intermedias ya establecidas por la unidad adquirida y a los activos intangibles de la economía receptora. Sería plausible pensar que cuanto más avanzado sea un SNI, mayor será la probabilidad de mejorar las relaciones usuario-productor, lo cual también favorecerá la competitividad y la innovación (Lundvall et al., 2002; Álvarez et al., 2009). Estos aspectos positivos no son tan inmediatos en el caso de la inversión greenfield debido a que no tiene por qué existir una relación previa entre la empresa inversora, los agentes nacionales y el tejido institucional.

Al tiempo, las FyA parecen estar negativamente relacionadas con el grado de apertura de los países de destino. Pese a no haber analizado explícitamente este aspecto como determinante de la forma de entrada, el signo negativo del coeficiente de apertura comercial puede relacionarse con los efectos derivados de las prácticas de tariff-jumping cuyo objetivo es evitar la protección comercial. Trabajos anteriores que analizan las diferencias en los determinantes de la IDE entre países muestran que, efectivamente, uno de los motivos de invertir en el exterior es la elusión de aranceles; dicho motivo explicaría las diferencias de la expansión de las empresas japonesas en los mercados internacionales vía greenfield, frente a las empresas europeas y estadounidenses que muestran una mayor inclinación a la inversión vía FyA (Belderbos et al., 2001).

En suma, el análisis empírico confirma satisfactoriamente la hipótesis planteada acerca de la evolución de las formas de entrada de IDE a lo largo de la última década. Se comprueba la existencia de factores tanto institucionales como económicos en los países receptores. Mientras que la presencia previa de capital extranjero es un factor determinante de las dos formas de entrada, las características institucionales de los SNI resultan ser un elemento más importante en las FyA. Las capacidades de absorción, por su lado, están más vinculadas a la adquisición de empresas con un mayor grado de integración en los sistemas productivos del país de destino. En este sentido, la significatividad de la intensidad en I+D no debe entenderse exclusivamente como un mero indicador de la capacidad de generación de conocimiento de los países sino como la expresión de sistemas tecnológicos y de producción más avanzados, en los que el marco institucional favorece las actividades innovadoras y productivas. Estos resultados pueden ser especialmente reveladores para el análisis de los países en desarrollo. Es plausible pensar que los cambios re- 
lacionados con una mayor estabilidad política y económica y con los esfuerzos destinados a activar los sistemas productivos y educativos favorecen la entrada de inversión vía FyA. Por lo tanto, la combinación de los diferentes aspectos señalados avala la idea de la existencia de una senda evolutiva de los flujos de IDE que puede describirse por medio del comportamiento de las inversiones extranjeras, del nivel de desarrollo de los SNI de los países y de la tendencia creciente a la internacionalización vía FyA.

\section{Conclusiones}

La importancia creciente que algunas economías en desarrollo están llegando a alcanzar en el comercio internacional y en la distribución de la IDE mundial, alienta a emprender nuevas investigaciones que combinen la perspectiva de internacionalización empresarial con aquella a nivel macro o a nivel de país. En este artículo se presenta un marco integrador de las formas de entrada de la IDE, proporcionando nueva evidencia empírica para una muestra amplia de países que incorpora economías desarrolladas y en desarrollo, desde la óptica del marco conceptual de los sistemas nacionales de innovación. Los resultados de nuestro análisis confirman que los factores que le son propios a sistemas más consolidados, explican mejor las FyA internacionales mientras que los factores económicos están más relacionados con las inversiones greenfield, confirmando la interacción existente entre formas de entrada y niveles de desarrollo. En particular, cabe destacar que los flujos de FyA están estrechamente relacionados con los aspectos más cualitativos del marco institucional de los países receptores, en tanto que la estabilidad institucional y las capacidades de absorción pueden ser elementos más atractivos para aquellas empresas con interés en compartir las actividades tecnológicas y las interacciones ya establecidas en el sistema productivo receptor. Aunque sigue existiendo una elevada concentración de IDE, los países en desarrollo han ido ganando terreno en la distribución de los flujos y su senda en relación a la IDE puede diferir respecto a los países más avanzados. En este sentido, para una mejor comprensión de las trayectorias, la evidencia que presentamos aquí lleva a subrayar el papel de las capacidades nacionales y el aprendizaje tecnológico sobre bases globales. Los resultados permiten ilustrar que existen comportamientos diferenciados según formas de entrada y que es notable la heterogeneidad de los países en desarrollo como economías receptoras de IDE, ya que las economías emergentes y los BRIC se encuentran contenidas en ese amplio grupo y condicionan un comportamiento claramente diferenciado. Los resultados invitan igualmente a ampliar la investigación sobre EMN, sus formas de entrada, el nivel de compromiso en los países receptores y su potencial impacto. 


\section{Referencias}

[1] AGARWARL, S. y S. RAMASWAMI (1992): Choice of foreign market entry mode: Impact of ownership, location and internationalization factors. Journal of International Business Studies, 23(1), pp. 1-27.

[2] ALDENDERFER, M.S. y R.K. BLASHFIELD (1984): Cluster Analysis. Series: Quantitative Applications in the Social Sciences. SAGE Publications, vol. 44.

[3] ÁLVAREZ, I. y G. MAGAÑA (2007): Technological development in middle-income countries, en: J.A. Alonso (Ed.), Cooperation with middle-income countries. Ed. Complutense, Madrid, pp. 339-369.

[4] ÁlVAREZ, I., R. MARÍN, y A. FONFRÍA (2009): The role of networking in the competitiveness of firms. Technological Forecasting \& Social Change, 76 (2009), pp. 410421.

[5] ARELLANO, M. y S.R. BOND (1991): Some tests of specification for panel data. Journal of Econometrics, 59, pp. 87-97.

[6] ARELLANO, M. y O. BOVER (1995): Another look at the instrumental variables estimation of error components models. Journal of Econometrics, 68, pp. 29-51.

[7] BAILEY, K.D. (1994): Typologies and Taxonomies: An introduction to classification techniques. Series: Quantitative Applications in the Social Sciences. SAGE Publications, vol. 102.

[8] BARKEMA, H.G. y F. VERMEULEN (1998): International expansion through start-up or acquisition: A learning perspective. Academy of Management Journal, 4(1), pp. 7-26.

[9] BARTLETT, C. A. y S. GHOSHAL (1998): Managing across borders: The transnational solution, Harvard Business School Press, Boston.

[10] BELDERBOS, R. (2003): Entry mode, organizational learning, and R\&D in foreign affiliates: evidence from Japanese firms. Strategic Management Journal, 24, pp. 235259.

[11] BELDERBOS, R.E., G. CAPANNELLI y K. FUKAO (2001): Backward vertical linkages of foreign manufacturing affiliates: Evidence from Japanese multinationals. World Development, 29(1), pp. 189-208.

[12] BERTRAND, O., K. HAKKALA y P.J. NORBÄCK (2007): Cross-border acquisition or greenfield entry: Does it matter for affiliate R\&D?. Research Institute of Industrial Economics, Working Paper Series, 693, Stockholm.

[13] BLUNDELL, R.W. y S.R. BOND (1998): Initial conditions and moment restrictions in dynamic panel models. Journal of Econometrics, 87, pp. 115-143.

[14] BROUTHERS, K.D. y L.E. BROUTHERS (2000): Acquisition or greenfield start-up? Institutional, cultural and transaction cost influences. Strategic Management Journal, 21, pp. 89-97.

[15] BROUTHERS, K.D. y L.E. BROUTHERS (2003): Why service and manufacturing entry mode choices differ: the influence of transaction cost factors, risk, and trust. Journal of Management Studies, 40, pp. 1179-204.

[16] CANTWELL, J. (1989): Technological Innovation and Multinational Corporations, Basil Blackwell, Oxford.

[17] CANTWELL, J. (1995): The globalization of technology: What remains of the product cycle model?, Cambridge Journal of Economics, 19(1), pp. 155-174. 
[18] CANTWELL, J. y J. MOLERO (2003): Multinational enterprises, innovative strategies and systems of innovation, Edward Elgar, Cheltenham.

[19] CANTWELL, J. y R. MUDAMBI (2005): MNE competence-creating subsidiary mandates. Strategic Management Journal, 26(12), pp. 1109-1128.

[20] CAPRON, L. (1999): The long-term performance of horizontal acquisitions. Strategic Management Journal, 20, pp. 987-1018.

[21] CHANG, S. J. y P.M. ROSENZWEIG (2001): The choice of entry mode in sequential foreign direct investment. Strategic Management Journal, 22(8), pp. 747-776.

[22] CHUNG, W. y J. ALCÁCER (2002): Knowledge seeking and location choice of foreign direct investment in the United States. Management Science, 48(12), pp. 1534-1554.

[23] COHEN, W.M. y D.A. LEVINTHAL (1990): Absorptive capacity: a new perspective on learning and innovation. Administrative Science Quarterly, 35, pp. 128-152.

[24] CUERVO-CAZURRA, A. (2007): Sequence of value-added activities in the multinationalization of developing country firms. Journal of International Management, 13 (2007), pp. 258-277.

[25] DAVIDSON, W.H. (1980): The location of foreign direct investment activity: country characteristics and experience effects. Journal of International Business Studies, 11(2), pp. 9-22.

[26] DAVIS, P.S., A.B. DESAI y J.D. FRANCIS (2000): Mode of international entry: An isomorphism perspective. Journal of International Business Studies, 31(2), pp. 239-258.

[27] DUNNING, J.H. (2001): The eclectic (OLI) paradigm of International production: Past, present and future. International Journal of the Economics of Business, 2001, vol. 8, pp. 173-190.

[28] DUNNING, J.H. (2006): Towards a paradigm of development: implications for the determinants of international business activity. Transnational Corporations, 15(1), pp. 173-227.

[29] DUNNING, J.H. y R. NARULA (Eds.) (1996): Foreign direct investment and governments: Catalysts for economic restructuring, Routledge, London.

[30] FREEMAN, C. (1987): Technology policy and economic performance: Lessons from Japan, Pinter, London.

[31] FROST, T. (2001): The geographic sources of foreign subsidiaries' innovations. Strategic Management Journal, 22, pp. 101-123.

[32] GLOBERMAN, S. y D. SHAPIRO (2002): Global foreign direct investment flows: the role of governance infrastructure. World Development, 30(11), pp. 1899-1919.

[33] HARZING, A.W. (1999): Acquisitions versus greenfield investments: International strategy and management of entry modes. Strategic Management Journal, 23, pp. 211-227.

[34] HITT, M.A., V. FRANKLIN y H. ZHU (2006): Culture, institutions and international strategy. Journal of International Management, 12, pp. 222-234.

[35] KAUFMANN, D., A. KRAAY y M. MASTRUZZI (2009): Governance Matters VIII: Aggregate and Individual Governance Indicators, 1996-2008 (June 29, 2009). World Bank Policy Research Working Paper No. 4978.

Disponible en SSRN: http://ssrn.com/abstract=1424591

[36] KETCHEN, D.J. y C.L. SHOOK (1996): The application of cluster analysis in strategic management research: an analysis and critique. Strategic Management Journal, 17, pp. 441-458. 
[37] KLINE, J. y N. ROSENBERG (1986): An overview of innovation, in: Landau, R. and N. Rosenberg (Eds.), The positive sum strategies: Harnessing technology for economic growth, National Academic Press, Washington DC.

[38] KOGUT, B. y H. SINGH (1988): The effect of national culture on the choice of entry mode. Journal of International Business Studies, 19(3), pp. 411-432.

[39] LALL, S. (2002): Implications of cross-border mergers and acquisitions by TNCs in developing countries: A beginners' guide. QEH Working Paper No. 88.

[40] LUNDVALL, B.A. (Ed.) (1992): National systems of innovation, Pinter Publishers, London.

[41] LUNDVALL, B.A., B. JOHNSON, E.S. ANDERSEN y B. DALUM (2002): National systems of production, innovation and competence building. Research Policy, 31, pp. 213 231.

[42] LUNDVALL, B.A., J. VANG, K.J. JOSEPH y C. CHAMINADE (2009): Bridging Innovation System Research and Development Studies: Challenges and research opportunities, Paper for the 7th Globelics Conference, Senegal, 6-8 October.

[43] MARÍN, R. e I. ÁLVAREZ (2009): Technological effects of M\&As in Spanish manufacturing, Industrial and Corporate Change, 18(4), pp. 761-785.

[44] MCCANN, P. y R. MUDAMBI (2005): Analytical differences in the economics of geography: the case of the multinational firm. Environment and Planning A, 37, pp. 1857-1876.

[45] MEYER, K.E. (2004): Perspectives on multinational enterprises in emerging economies. Journal of International Business Studies, 35(4), pp. 259-276.

[46] MOWERY, D.C. y E. OXLEY (1995): Inward technology transfer and competitiveness: the role of national innovation systems. Cambridge Journal of Economics, 19(1), pp. 67-93.

[47] MUDAMBI, R. (1995): The MNE investment location decision: some empirical evidence. Managerial and Decision Economics, 16, pp. 249-257.

[48] MUDAMBI, R. (2002): Knowledge management in multinational firms. Journal of International Management, 8, pp. 1-9.

[49] MUDAMBI, R. (2008): Location, control and innovation in knowledge-intensive industries. Journal of Economic Geography, 8, pp. 699-725.

[50] MUDAMBI, R. y S.M. MUDAMBI (2002): Diversification and market entry choices in the context of foreign direct investment. International Business Review, 11, pp. 35-55.

[51] NARULA, R. (1996): Multinational investments and economic structure, Routledge, London.

[52] NARULA, R., P. CRISCUOLO y B. VERSPAGEN (2002): The relative importance of home and host innovation systems in the internationalisation of MNE R\&D: a patent citation analysis. Merit Memorandum MERIT RM 2002-26.

[53] NARULA, R. y J.H. DUNNING (2000): Industrial development, globalisation and multinational enterprises: new realities for developing countries. Oxford Development Studies, 28(2), pp. 141-167.

[54] NELSON, R. (1993): National innovation systems: a comparative analysis, Oxford University Press, Oxford.

[55] OZAWA, T. (1992): Foreign direct investment and economic development. Transnational Corporation, 1(1), pp. 27-54. 
[56] PEARCE, R. (1999): Decentralised R\&D and strategic competitiveness: Globalised approaches to generation and use of technology in multinational enterprises (MNEs). Research Policy, 28, pp. 157-178.

[57] PEARCE, R. (2006): Globalization and development: an international business strategy approach. Transnational Corporations, 15(1), pp. 39-74.

[58] PISCITELLO, L. (2004): Corporate diversification, coherence and economic performance. Industrial and Corporate Change, 13(5), pp. 757-787.

[59] PORTER, M. E. (1986): Changing patterns of international competition. California Management Review, 28(2), pp. 9-40.

[60] ROSENZWEIG, P.M. y J.V. SINGH (1991): Organizational environments and the multinational enterprise.The Academy of Management Review, 16(2), pp. 340-361.

[61] RUGMAN, A.M. y J.P. DOH (2008): Multinationals and development, Yale University Press, New Haven and London.

[62] RUGMAN, A.M. y A. VERBEKE (2001): Location, competitiveness and the multinational enterprise, in: Rugman, A.M. and T.L. Brewer (Eds.), The Oxford Handbook of International Business, Oxford University Press, Oxford.

[63] SANNA-RANDACCIO, F. y R. VEUGELERS (2007): Multinational knowledge spillovers with decentralised R\&D: a game-theoretic approach. Journal of International Business Studies, 38(1), pp. 47-63.

[64] SANTISO, J. (2008): La emergencia de las multilatinas, Revista de la CEPAL, 95 (Agosto), pp. 7-30.

[65] SCOTT, W.R. (1981): Organizations: Rational, natural, and open systems, Englewood Cliffs, Prentice-Hall, NJ.

[66] SCOTT, W.R. (1983): The organization of environments: Network, cultural, and historical elements, en: Meyer, J.W. y W.R. Scott (Eds.), Organizational environments, Sage, London, pp. 155-175.

[67] SHIMIZU, K., M.A. HITT, D. VAIDYANATH y V. PISANO (2004): Theoretical foundations of cross-border mergers and acquisitions: A review of current research and recommendations for the future. Journal of International Management, 10, pp. 307-355.

[68] SINGH, J. (2007): Asymmetry of knowledge spillovers between MNCs and host country firms. Journal of International Business Studies, 38, pp. 764-786.

[69] SLANGEN, A. y J.F. HENNART (2007): Greenfield or acquisition entry: a review of the empirical foreign establishment mode literature. Journal of International Management, 13, pp. 403-429.

[70] TIHANYI, L. y A.S. ROATH (2002): Technology transfer and institutional development in Central and Eastern Europe. Journal of World Business, 37, pp. 188-198.

[71] UNCTAD (2005): World Investment Report: Transnational corporations and the internationalization of R\&D, United Nations, New York and Geneva.

[72] UNCTAD (2007): World Investment Report: Transnational corporations, extractive industries and development, United Nations, New York and Geneva.

[73] VON HIPPEL, E. (1988): The sources of innovation, Oxford University Press, Oxford.

[74] XU, B. (2000): Multinational enterprises, technology diffusion, and host country productivity growth. Journal of Development Economics, 62, pp. 477-493.

[75] YANG, Q., R. MUDAMBI y K.E. MEYER (2008): Conventional and reverse knowledge flows in multinational corporations. Journal of Management, 34(5), pp. 882-902. 


\section{ANEXO}

CUADRO A1

DESCRIPCIÓN DE LAS VARIABLES

\begin{tabular}{|c|c|c|}
\hline Variable & Definición & Fuente \\
\hline IDE & $\begin{array}{l}\text { Inversión directa extranjera (flujos netos, dólares EEUU } \\
\text { constantes, 2000), en logaritmos naturales }\end{array}$ & $\begin{array}{l}\text { UNCTAD, } \\
\text { FDI database }\end{array}$ \\
\hline FyA & $\begin{array}{l}\text { Fusiones y adquisiciones (flujos, dólares EEUU } \\
\text { constantes, 2000), en logaritmos naturales }\end{array}$ & $\begin{array}{l}\text { UNCTAD, } \\
\text { FDI database }\end{array}$ \\
\hline IDEStock & $\begin{array}{l}\text { Stock de IDE (dólares EEUU constantes, 2000), } \\
\text { en logaritmos naturales }\end{array}$ & $\begin{array}{l}\text { Banco Mundial, } \\
\text { WDI } 2007\end{array}$ \\
\hline ID & $\begin{array}{l}\text { Gasto en I+D (como porcentaje del PIB), } \\
\text { en logaritmos naturales }\end{array}$ & $\begin{array}{l}\text { Banco Mundial, } \\
\text { WDI } 2007\end{array}$ \\
\hline GMI & Índice de gobernabilidad & Banco Mundial \\
\hline PIB & $\begin{array}{l}\text { Producto interior bruto (dólares EEUU constantes, } \\
\text { 2000), en logaritmos naturales }\end{array}$ & $\begin{array}{l}\text { Banco Mundial, } \\
\text { WDI } 2007\end{array}$ \\
\hline$\Delta \mathrm{PIB}$ & $\begin{array}{l}\text { Tasa de crecimiento anual del PIB, en logaritmos } \\
\text { naturales }\end{array}$ & $\begin{array}{l}\text { Banco Mundial, } \\
\text { WDI } 2007\end{array}$ \\
\hline AP & $\begin{array}{l}\text { Grado de apertura: exportaciones e importaciones } \\
\text { de bienes y servicios como porcentaje del PIB, } \\
\text { en logaritmos naturales }\end{array}$ & $\begin{array}{l}\text { Banco Mundial, } \\
\text { WDI } 2007\end{array}$ \\
\hline W & $\begin{array}{l}\text { Sueldos y salarios (dólares EEUU constantes, 2000), } \\
\text { en logaritmos naturales }\end{array}$ & $\begin{array}{l}\text { Banco Mundial, } \\
\text { WDI } 2007\end{array}$ \\
\hline CK & $\begin{array}{l}\text { Capital humano: matriculados en educación secundaria } \\
\text { como porcentaje de la población total en edad de cursar } \\
\text { estudios secundarios, en logaritmos naturales }\end{array}$ & $\begin{array}{l}\text { Banco Mundial, } \\
\text { WDI } 2007\end{array}$ \\
\hline
\end{tabular}

